\title{
Erythromycin attenuates an experimental model of chronic bronchiolitis via augmenting monocyte chemoattractant protein-1
}

\author{
T. Takahashi*,\#, M. Suga*, A. Matsukawa\# ${ }^{\#}$ K. Sato*, T. Okamoto*, H. Ichiyasu*, S. Ohkawara\#, \\ M. Yoshinaga\#, M. Ando*
}

Erythromycin attenuates an experimental model of chronic bronchiolitis via augmenting monocyte chemoattractant protein-1. T. Takahashi, M. Suga, A. Matsukawa, K. Sato, T. Okamoto, H. Ichiyasu, S. Ohkawara, M. Yoshinaga, M. Ando. C) ERS Journals Ltd 2001.

ABSTRACT: The mechanisms underlying the therapeutic efficacy of erythromycin (EM) in diffuse panbronchiolitis (DPB) was investigated. For this purpose, an experimental rabbit model of DPB induced by Pseudomonas aeruginosa inoculation was employed. Daily administration of EM $\left(3 \mathrm{mg} \cdot \mathrm{kg} \cdot \mathrm{day}^{-1}\right)$ led to an increase in the number of macrophages in bronchoalveolar lavage fluid (BALF) at an early phase, while reducing the size of granulomatous lesions at the late phase without affecting the number of viable bacteria recovered from the infected lung.

Reverse transcriptase polymerase chain reaction (RT-PCR), enzyme-linked immunosorbent assay (ELISA) and immunohistochemical studies showed that monocyte chemoattractant protein (MCP)-1 was produced in both BALF and infected lung. EM treatment resulted in a significant increase in the level of MCP-1 in BALF, while reducing that of tumour necrosis factor (TNF)- $\alpha$, interleukin (IL)-1 $\beta$ and IL-8. EM also increased MCP-1 messenger ribonucleic acid (mRNA) and protein expression in the infected lung. MCP-1 blockade abolished the protective effect of EM, as neutralization of MCP-1 with anti-MCP-1 antibodies reduced the EM-induced increase in the number of macrophages in BALF, and augmented size of the granulomatous lesions, as compared to control.

The results of the present study suggest that erythromycin attenuates the pulmonary granuloma formation, at least in part, by increasing the production of monocyte chemoattractant protein-1.

Eur Respir J 2001; 17: 360-367.
*First Dept of Internal Medicine and \#First Dept of Pathology, Kumamoto University School of Medicine, Honjo, Kumamoto 860-0811, Japan.

Correspondence: T. Takahashi, First Dept of Internal Medicine, Kumamoto University School of Medicine, 1-1-1, Honjo, Kumamoto 860-0811, Japan. Fax: 81963710582

Keywords: Bronchiolitis erythromycin

monocyte chemoattractant protein-1 Pseudomonas aeruginosa

Received: March 92000

Accepted after revision September 20 2000

This study was funded by a grant from The Japanese Ministry of Education, Science, Sports and Culture, Japan.
Diffuse panbronchiolitis (DPB) is a chronic infectious airway disease with high morbidity and mortality $[1,2]$. However, the prognosis for DPB has dramatically improved over the past decade with the use of erythromycin (EM) $[3,4]$. The efficacy of EM in the treatment of DPB does not appear to depend on its original antimicrobial activity, as levels of EM in the serum and sputum after EM treatment are lower than the minimum inhibitory concentrations for Haemophilus influenzae and Pseudomonas aeruginosa, which can be isolated from the sputum of patients with DPB [5]. The viable bacteria is frequently recovered from the sputum in EM responsive patients [5].

Recent studies have suggested that the beneficial effect of EM is due to its anti-inflammatory properties [4-6]. In vitro, EM inhibited the production of pro-inflammatory cytokines such as tumour necrosis factor (TNF)- $\alpha$, interleukin (IL)-1 $\beta$ and IL-8 [7-9], all of which were found in bronchoalveolar lavage fluid (BALF) of DPB patients and are regarded as being key mediators in the pathogenesis of DPB $[10,11]$. EM treatment significantly reduced the levels of IL-1 $\beta$ and
IL-8 in BALF of DPB patients, which was accompanied by a decreased number of neutrophils in BALF $[10,11]$. Thus, it appears that EM inhibits neutrophil influx, a prominent feature in BALF of DPB patients, by suppressing pro-inflammatory cytokines.

Another distinctive pathologic feature of DPB is the accumulation of foamy alveolar macrophages in the lung $[1,2]$, suggesting that mediators known to attract monocytes may be involved in the progression of DPB. Recently, a family of proteins with specific chemotactic activity for monocytes, CC chemokines, has been identified. Monocyte chemoattractant protein (MCP)-1 is a prototype of CC chemokines, and has been detected in clinical diseases characterized by a massive macrophage influx [12, 13]. However, little is known about the role of MCP-1 in the development of either clinical DPB or experimental models. It is possible that MCP-1 is involved in DPB, and EM may have a role in regulating the production/function of MCP-1.

In the present study, attempts to elucidate the effects of EM on the recruitment of macrophages and 
production of MCP-1 in DPB were made. For this purpose, an experimental model of chronic bronchiolitis induced by $P$. aeruginosa inoculation was employed [14]. The results show that EM treatment ameliorates granuloma formation via up-regulating MCP-1. This novel activity of EM may be one of the mechanisms underlying the therapeutic efficacy of EM in the treatment of DPB.

\section{Materials and methods}

Animals

Male Japanese white rabbits $(2.0-2.5 \mathrm{~kg}$; Inoue Laboratory Animals, Kumamoto, Japan) were housed under standard care. Guidelines and permission for performance of all the animal experiments was provided by the Animal Care Committee of Kumamoto University Medical School.

\section{Pseudomonas aeruginosa}

EM resistant mucoid $P$. aeruginosa was isolated from human otorrhoea cultures, and enmeshed in agar beads, as described [14]. An aliquot of the agar beads containing $P$. aeruginosa was homogenized, serially diluted with sterile saline, loaded on agar plates (NAC, Nissui Pharmaceutical, Tokyo, Japan), and the colony forming units (CFU) were determined.

\section{In vivo experiments}

Rabbits were anesthetized with pentobarbital sodium $\left(30 \mathrm{mg} \cdot \mathrm{kg}^{-1}\right.$, i.v. $)$, and $2 \mathrm{~mL}$ of agar beads suspension containing $1 \times 10^{7} \mathrm{CFU}$ of $P$. aeruginosa was intratracheally inoculated. Erythromycin lactobionate (Dainippon Pharmaceutical, Osaka, Japan) or saline was administered i.v. immediately and every $24 \mathrm{~h}$ after the inoculation until sacrifice $\left(3 \mathrm{mg} \cdot \mathrm{kg} \cdot \mathrm{day}^{-1}\right)$, according to the previous report [15]. The dosage was determined by preliminary studies, showing that $3 \mathrm{mg} \cdot \mathrm{kg} \cdot \mathrm{day}^{-1}$ of EM was most effective for the treatment of the present model. To neutralize MCP-1 activity, $1 \mathrm{~mL}$ of anti-MCP-1 antiserum was administered $i . v$. immediately and every 2 days after the inoculation. The neutralizing antirabbit MCP-1 antibodies were raised by immunizing a goat with rabbit MCP-1, as described [16]. The volume of antiserum was considered to be sufficient, because the half-life of the antibodies in the circulation was $48 \mathrm{~h}$ and a larger volume $(3 \mathrm{~mL})$ did not induce further effects. As a control, pre-immune goat serum was used. The endotoxin content in the sera was below the detection level $\left(<0.05 \mathrm{EU} \cdot \mathrm{mL}^{-1}\right.$, Pyrogent; BioWhittaker, Walkersville, MD, USA).

At appropriate time intervals after $P$. aeruginosa inoculation, the rabbits were anaesthetized, bled, euthanized, and both lungs were resected. The right lung was lavaged with $3 \times 25 \mathrm{~mL}$ sterile saline, centrifuged at $500 \times g$ for $10 \mathrm{~min}$ at $4^{\circ} \mathrm{C}$, and the supernatants $(\mathrm{BALF})$ were stored at $-30^{\circ} \mathrm{C}$ for later assay.
The cell pellets were resuspended in $1 \mathrm{~mL}$ saline, and the number of leukocytes counted using a haemocytometer. Differential cell analyses were performed by Diff-Quik stained cytospin preparations [17]. The lower lobe of the left lung was weighed and frozen in liquid nitrogen to determine the cytokine levels. The upper lobe of the left lung was fixed with $4 \%$ paraformaldehyde, and used for histological examinations. For the bacteriological analysis, both lungs were homogenized using a polytron AG homogenizer (Kinematica Instruments, Lucern, Switzerland). The homogenates were serially diluted, plated on NAC agar plates, cultured overnight at $37^{\circ} \mathrm{C}$, and the $\mathrm{CFU}$ were determined. Preliminary studies showed that the pulmonary lesions equally distributed in the lung after the intratracheal inoculation of $P$. aeruginosa, as histological analyses and cytokine levels between different parts of the lung were similar.

\section{Study design}

In order to determine the natural course in this model, $P$. aeruginosa was inoculated in the lung, and the infected lungs were examined histologically on day 0,1 , 3, 14 (six rabbits each, total of 24 rabbits). An additional six rabbits were killed at $18 \mathrm{~h}$ after the inoculation, and messenger ribonucleic acid (mRNA) expression for MCP-1 was determined by the leukocytes harvested from the BALF. Control cells were harvested from the BALF of normal rabbits (six rabbits). To assess the effects of EM on the recruitment of leukocytes and cytokine levels in the BALF, either EM or vehicle was i.v. administered after the inoculation of $P$. aeruginosa, and the samples were harvested on day $0,1,3,7$ and 14 (six rabbits in both groups at each time-point, total of 60 rabbits). The different sets of rabbits were used for histological analyses, which were harvested on day 1, 3, 7 and 14 (six rabbits in both groups, total of 48 rabbits). In order to examine the involvement of MCP-1 in this model, rabbits infected with $P$. aeruginosa were treated with erythromycin plus either anti-MCP-1 or control serum, and the BALF samples were harvested on day 3 and 14 (six rabbits in both groups at each time-point, total 24 rabbits). The different sets of 24 rabbits were used for histological analyses. The total number of rabbits used in this study was 192.

\section{Assessment of granulomatous lesions in the lung}

The lung sections were stained with haematoxylin and eosin. The granulomatous lesions (a minimum of 10 lesions) were randomly chosen, and captured with an NIH image (a public domain image processing and analysis programme by the National Institutes of Health image). The area was converted from pixels ${ }^{2}$ to $\mathrm{mm}^{2}$ by measuring a known area on a haemocytometer grid.

\section{Preparation of tissue extracts}

Freshly isolated lung tissues $(1 \mathrm{~g})$ were placed in $5 \mathrm{~mL}$ of homogenization buffer $(50 \mathrm{mM}$ phosphate 
buffer (PBS) pH 6.0, containing 0.5\% hexadecyltrimethylammonium bromide (Sigma Chemical, St. Louis, MO, USA), $1 \mathrm{mM}$ phenyl methyl sulphonil fluoride (Sigma), $20 \mathrm{mM}$ EP 475 (Taisho Pharmaceutical, Ohmiya, Japan), and $0.1 \mu \mathrm{g} \cdot \mathrm{mL}^{-1}$ bestatin (Sigma)) and homogenized. The homogenates were then sonicated, centrifuged at $15,000 \times g$ for $30 \mathrm{~min}$, and the resultant cleared supernatants were dialyzed against phosphate-buffered saline (PBS) and used for cytokine measurements.

\section{Measurement of cytokines}

Protein concentrations of MCP-1, IL-1 $\beta$ and IL-8 were measured, as previously described [16, 18, 19]. TNF- $\alpha$ activity was determined by L929 cell cytotoxic assay $[19,20]$. Detection limits were $3 \mathrm{pg} \cdot \mathrm{mL}^{-1}$ for MCP-1, $10 \mathrm{pg} \cdot \mathrm{mL}^{-1}$ for IL-1 $\beta, 30 \mathrm{pg} \cdot \mathrm{mL}^{-1}$ for IL-8 and $\mathrm{TNF}-\alpha$, respectively. Each detection system did not cross-react with other cytokines.

\section{Immunohistochemistry}

After blocking of endogenous peroxidase with $0.3 \%$ $\mathrm{H}_{2} \mathrm{O}_{2}$ in methanol, the tissue sections were treated with $0.05 \%$ trypsin for $10 \mathrm{~min}$ at room temperature, and then incubated overnight with $5 \mu \mathrm{g} \cdot \mathrm{mL}^{-1}$ of polyclonal anti-MCP-1 goat immunoglobulin Ig-G at $4{ }^{\circ} \mathrm{C}$. Preimmune goat IgG was used as a control. The sections were then rinsed and incubated for $30 \mathrm{~min}$ with $5 \mu \mathrm{g} \cdot \mathrm{mL}^{-1}$ of biotinylated rabbit antigoat $\mathrm{IgG}$ (Vector Laboratories, Burlingame, CA, USA). After washing, the sections were incubated with avidin-biotinperoxidase complex (Vector) for $30 \mathrm{~min}$ at room temperature. As a chromogen, 3,3'-diaminobenzidine (Sigma) was used. Haematoxylin was used for counter staining.

\section{Reverse transcriptase polymerise chain reaction}

RNA was isolated from samples using a MicroFastTrack $^{\mathrm{TM}}$ Kit (Invitrogen, San Diego, CA, USA), and reverse-transcribed to complementary deoxyribose nucleic acid (cDNA) with Oligo (dT) as primers (Gibco-BRL, Rockville, MD, USA). First-strand cDNAs were then amplified in the presence of Taq polymerase (Takara Shuzo, Shiga, Japan) and specific primers. The primers were designed to amplify rabbit MCP-1 and glyceraldehyde-3-phosphate dehydrogenase (GAPDH). MCP-1, sense primer: 5'-ATGAAGGTCTCTGCAACGCTTCTG-3'; antisense primer: 5' -TTACAATGAAGTAGTAGTAGAGGGTGT-3'. GAPDH: sense primer; 5'-GATCCATTCATTGACC TCC-3'; antisense primer: 5'-GATCTCGCTCCTGGAAGATG-3'. The polymerase chain reaction (PCR) was carried out at 35 cycles of $94^{\circ} \mathrm{C}$ for $1 \mathrm{~min}, 55^{\circ} \mathrm{C}$ for $1 \mathrm{~min}, 72^{\circ} \mathrm{C}$ for $2 \mathrm{~min}$. Ten microlitres of PCR products were subjected to electrophoresis on a $2 \%$ agarose gel in the presence of ethidium bromide.

\section{Statistical analysis}

Statistical significance of the data was determined by analysis of variance (ANOVA). A p-value $<0.05$ was considered to be statistically significant. All data are expressed as mean \pm SEM.

\section{Results}

Effects of erythromycin on the recruitment of leukocyte populations in bronchoalveolar lavage fluid

Inoculation of $P$. aeruginosa induced a massive leukocyte infiltration in BALF. Most of the leukocytes in BALF were macrophages, which peaked on day 1 (fig. 1). Once they decreased on day 3 , the number of macrophages increased again with time for up to 14 days. The recruitment of neutrophils peaked on day 1 and then decreased gradually (fig. 1). EM treatment dramatically increased the number of macrophages in BALF on day 3, while inhibiting on day 14 (fig. 1). EM inhibited the recruitment of neutrophils throughout the observation periods after day 3 (fig. 1). A small number of lymphocytes was seen in the BALF, which was unchanged by EM treatment (data not shown). Agar beads alone did not cause infiltration of a significant number of leukocytes. Thus, EM altered the recruitment of macrophages as well as neutrophils in BALF.

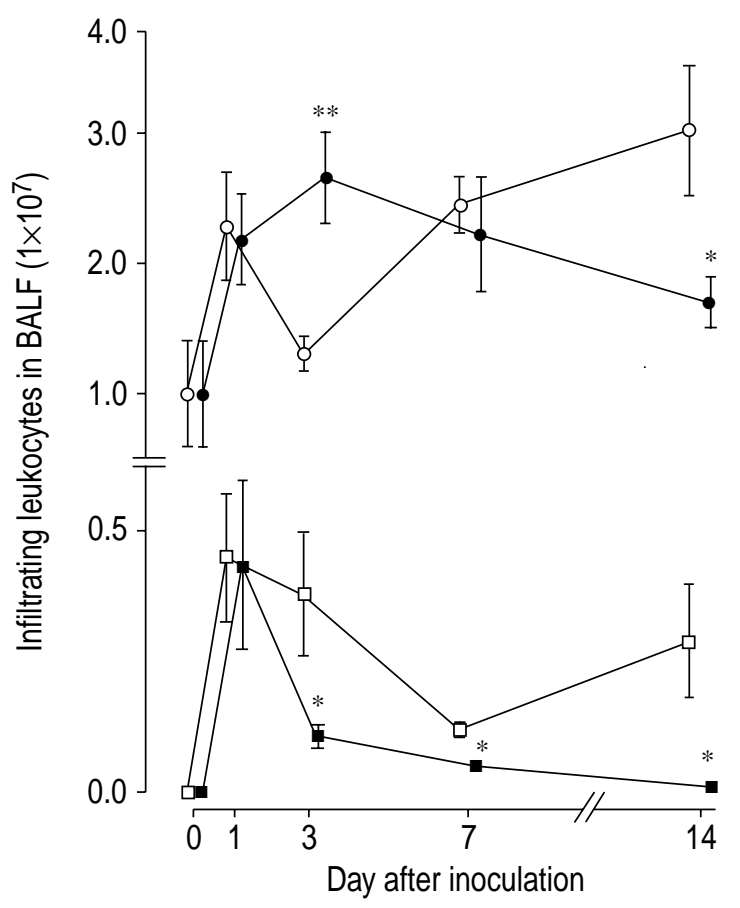

Fig. 1. - Effects of erythromycin on the recruitment of leukocytes in bronchoalveolar lavage fluid after the inoculation of $P$. aeruginosa. The number of macrophages $(\bullet)$ and neutrophils (ם) after treatment with erythromycin, and the number of macrophages $(\bigcirc)$ and neutrophils $(\square)$ after saline treatment are shown. Data represent the mean \pm SEM. *: $\mathrm{p}<0.05$; **: $\mathrm{p}<0.01$; when compared to the saline control. 
(a)

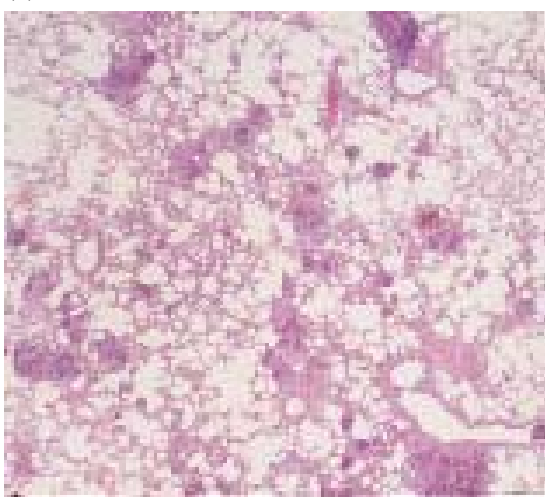

(d)

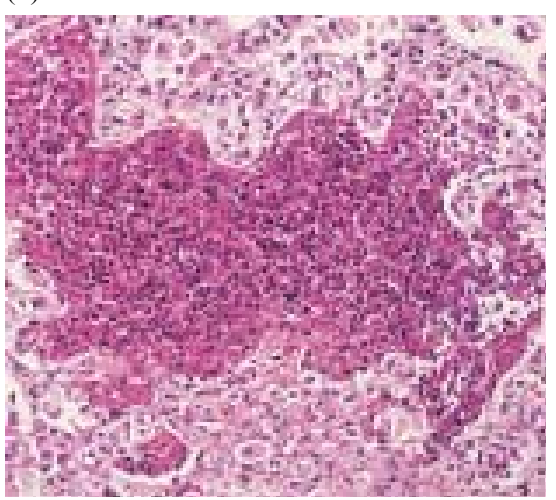

(b)

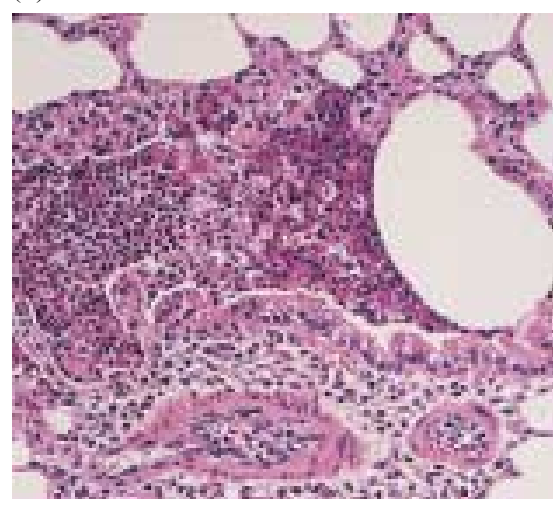

(e)

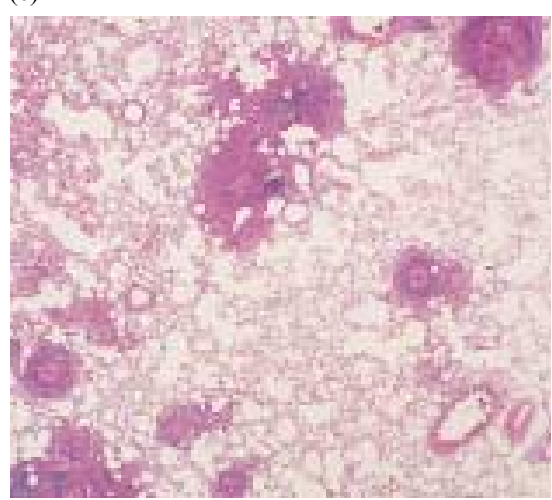

(c)

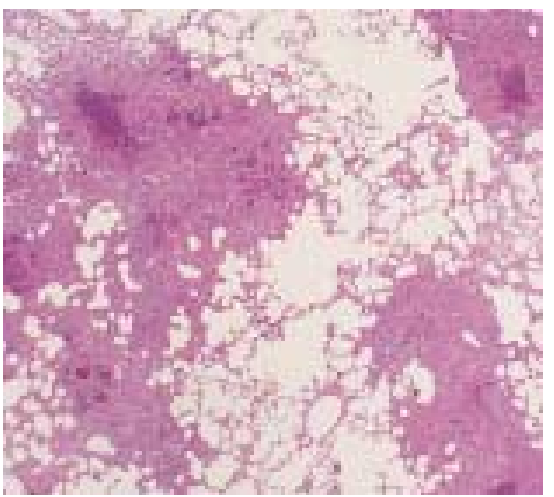

(f)

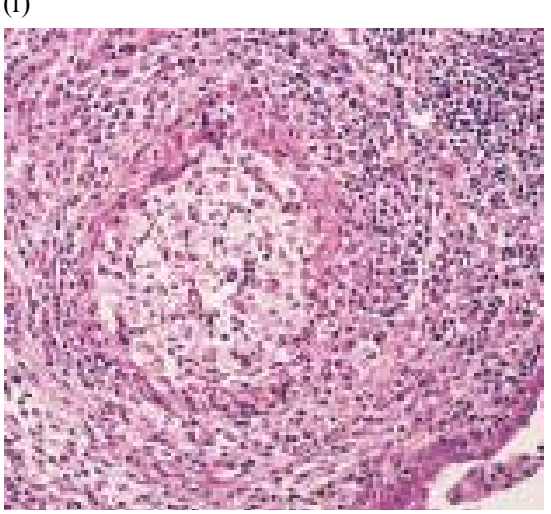

Fig. 2. - Histology of the lung after inoculation of $P$. aeruginosa. The representative photographs from six rabbits in the lung section are shown. a) and b) Day 1; c) and d) day 3; e) and f) day 14. Original magnification: a, c, e $\times 20$; b, d, f $\times 200$.

\section{Effects of erythromycin on granuloma formation}

The histological findings in the infected lung are shown in figure 2. The neutrophil infiltration was evident in peripheral bronchial walls and air spaces on day 1 (figs. 2a and b), which significantly increased on day 3 and formed granulomatous lesions (figs. 2c and d). Leukocyte populations changed thereafter, and macrophages and lymphocytes were predominant in the granulomatous lesions. On day 14, the granulomatous lesions became fully matured granulomas consisting of foamy macrophages and lymphocytes (figs. 2e and f). These findings resembled those seen in the lung of DPB patients. Lungs inoculated with agar beads alone showed little inflammatory reactions (data not shown). The area of the granulomatous lesions in the lung is shown in figure 3, which demonstrated that it peaked on day 3 and decreased gradually thereafter. Administration of EM resulted in a decrease in the size of granulomatous lesions by $50 \%$ on day 14 , as compared to the control, although no difference was found for the first 7 days (fig. 3). Thus, a chronic bronchiolitis, resembling clinical DPB, was induced in this model, and the granuloma formation was inhibited by EM treatment.

\section{Effects of erythromycin on the bacterial load}

$P$. aeruginosa used in this study was resistant to EM in vitro. The bacterial load in this model was also examined, which showed that EM did not affect the viable bacteria recovered from the lungs (EM versus control: day $3: 3.3 \pm 2.1$ versus $1.4 \pm 0.2 \times 10^{4} \mathrm{CFU}$; day $7: 7.5 \pm 2.3$ versus $5.6 \pm 0.7 \times 10^{4} \mathrm{CFU}$; day $14: 1.8 \pm 0.9$ versus $1.3 \pm 0.5 \times 10^{4} \mathrm{CFU}$; each group contained six rabbits; all the data was not significant). Thus, EM failed to clear bacteria from the infected lung.

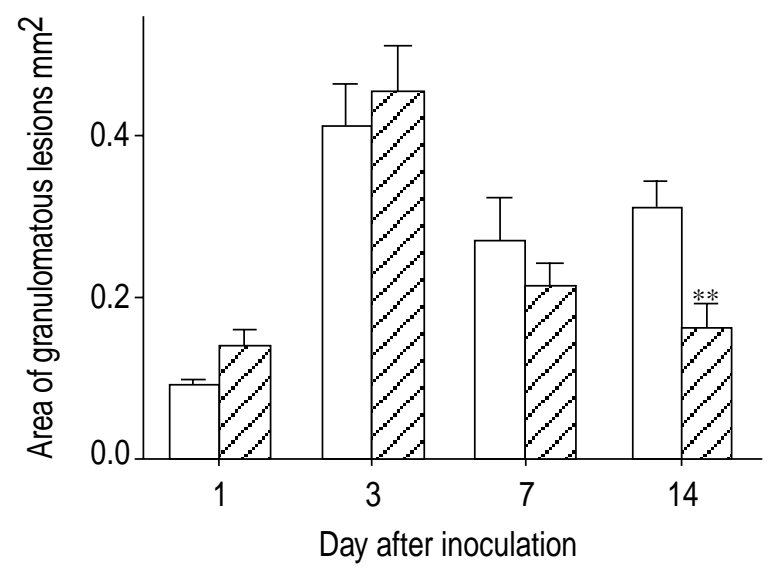

Fig. 3. - Effects of erythromycin on the area of granulomatous lesions after inoculation of $P$. aeruginosa. Rabbits were treated with: $\mathbb{Z}$ : erythromycin; or $\square$ : saline; and the granuloma size in the lungs was assessed. Data represent the mean \pm SEM. **: $\mathrm{p}<0.01$ when compared to saline control. 
a)



b)

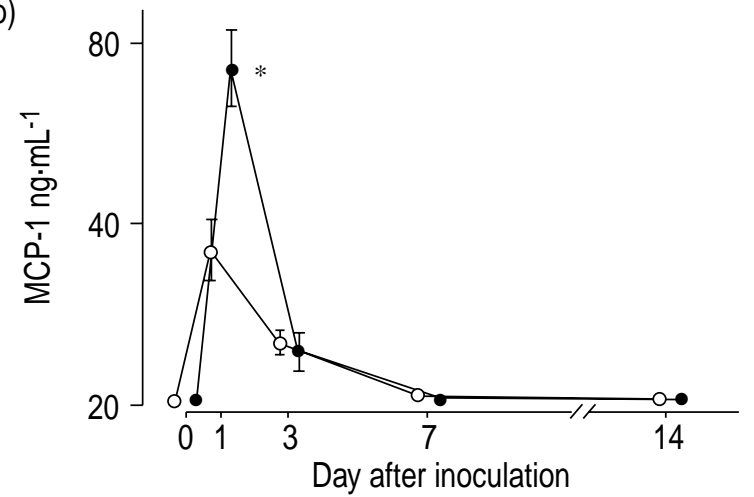

c) EM treatment

MCP-1

GAPDH

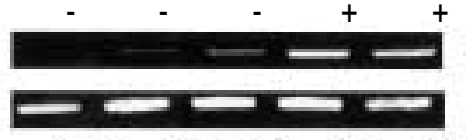

control exp. 1 exp. 2 exp. 1 exp.2
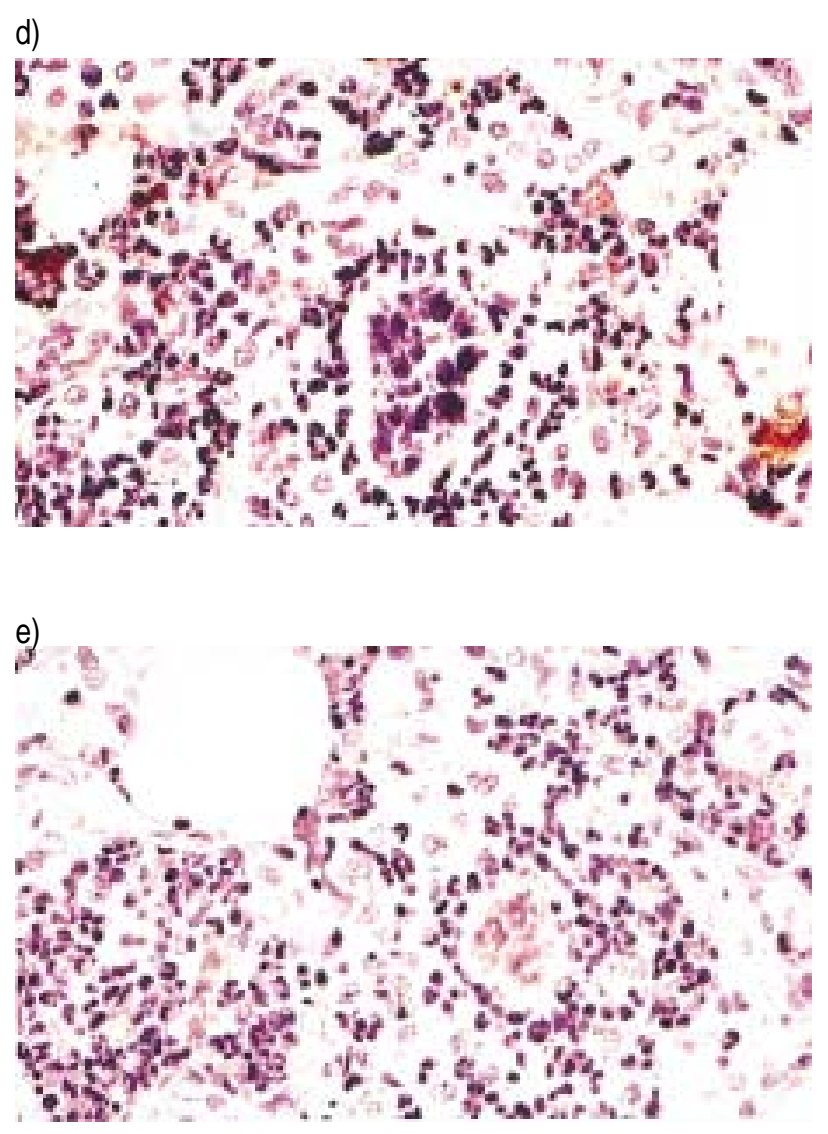

Fig. 4. - Effects of erythromycin (EM) on the production of monocyte chemoattractant protein (MCP)-1 after inoculation of $P$. aeruginosa. The levels of MCP-1 in: the a) bronchoalveolar lavage fluid (BALF) and b) lung after treatment with erythromycin $(\bullet)$ or saline $(\bigcirc)$. Data represent the mean $+\mathrm{SEM}$. *: $\mathrm{p}<0.05 ; * *: \mathrm{p}<0.01$; when compared to the saline control. c) Representative reverse transcriptase polymerase chain reaction (RT-PCR) analyses showing the expression of MCP-1 messenger ribonucleic acid in leukocytes in BALF at $18 \mathrm{~h}$ after the inoculation of $P$. aeruginosa. Each PCR was run in duplicate. The lung sections obtained on day 1 were stained with anti-MCP-1 immunoglobulin-(Ig)G or pre-immune IgG: d) positive; e) negative (GAPDH: reduced glyceraldehyde-phosphate dehydrogenase. original magnification, $\times 350)$.

\section{Effects of erythromycin on cytokine production}

Since macrophages were the major cells found in BALF and EM treatment altered the recruitment of macrophages, experiments were conducted next to measure the level of MCP-1, a potent monocyte chemoattractant. The levels of MCP-1 in both BALF and lung increased rapidly after $P$. aeruginosa inoculation, which peaked on day 1 , and then decreased gradually and returned to a basal level by day 7 (figs. 4a and b). Immunohistochemistry revealed that MCP-1 was expressed in macrophages and neutrophils (fig. 4d). Interestingly, peak levels of MCP-1 in both BALF and lung were significantly increased by EM treatment (figs. 4a and b). Likewise, MCP-1 mRNA expression in the leukocytes recovered from BALF was up-regulated by the treatment (fig. $4 \mathrm{c}$ ).

The levels of TNF- $\alpha$, IL- $1 \beta$ and IL- 8 were also measured in this model. EM treatment significantly inhibited the levels of TNF- $\alpha$ and IL-8 in BALF, when compared to the control (figs. 5a and c). Although IL-1 $\beta$ in BALF was unchanged by EM at the peak (day 1), no IL-1 $\beta$ was detected on day 3 after EM treatment whereas an appreciable level of IL-1 $\beta$ was present in control BALF (fig. 5b). The data indicate that MCP-1 was temporally produced in this model, and EM augmented the production of MCP-1 while reducing the production of TNF- $\alpha$, IL-1 $\beta$ and IL- 8 .

Effects of erythromycin are abolished by monocyte chemoattractant protein-1 blockade

To assess the involvement of MCP-1 in the therapeutic effects of EM in this model, rabbits were treated with EM plus either anti-MCP-1 antiserum or control serum after $P$. aeruginosa inoculation. As shown in figure $6 \mathrm{a}$, anti-MCP-1 antibodies significantly decreased the number of macrophages, but not neutrophils and lymphocytes, in BALF on day 3 as compared to the control. At this time point, the size of the pulmonary granulomatous lesions was unchanged (data not shown). However, anti-MCP-1 treatment significantly augmented the size of the lesions on day 14 

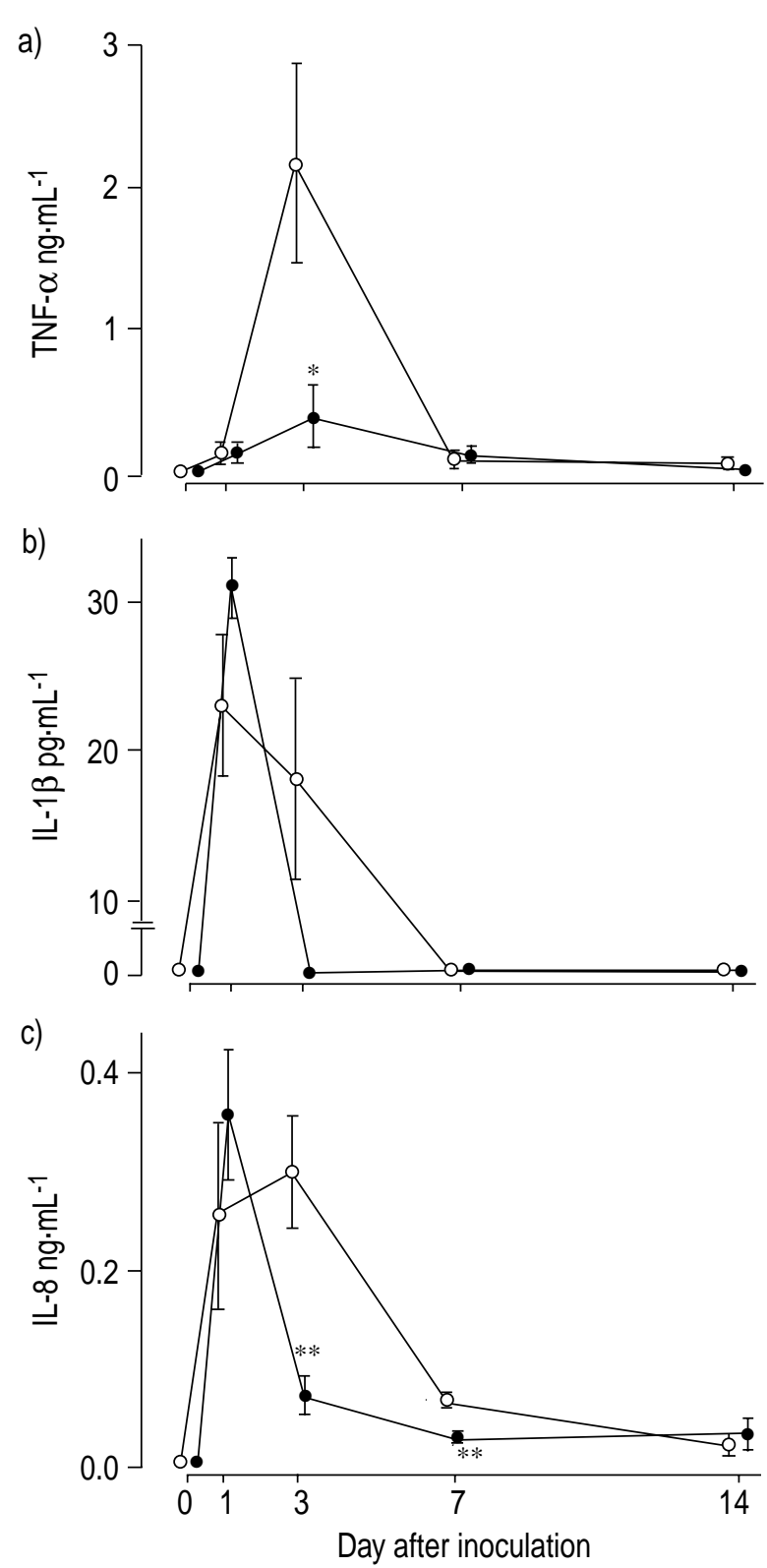

Fig. 5. - Effects of erythromycin on the production of tumour necrosis factor (TNF)- $\alpha$, interleukin (IL)-1 $\beta$ and interleukin (IL)- 8 in the bronchoalveolar lavage fluid. a) The levels of TNF- $\alpha$; b) IL$1 \beta$; and c) IL-8 in the BALF after the inoculation of $P$. aeruginosa were measured after the treatment with erythromycin $(\bullet)$ or saline $(\bigcirc)$. Data represent the mean \pm SEM. *: $\mathrm{p}<0.05 ; * *$ : $\mathrm{p}<0.01$; when compared to the saline control.

as compared to control (fig. 6b), without affecting the number of leukocyte populations in BALF (data not shown). The levels of TNF- $\alpha$, IL- $1 \beta$ and IL- 8 in BALF were not altered by anti-MCP-1 antibodies, at any time examined (data not shown). The bacterial load in the lung on day 7 was unchanged by anti-MCP-1 treatment (EM plus anti-MCP-1 antiserum versus EM plus control serum: $5.6 \pm 5.7$ versus $4.6 \pm 2.2 \times 10^{4}$ CFU, each group contained six rabbits and the data was not significant). Thus, MCP-1 blockade abolished the protective effects of EM in the regulation of granuloma formation in this model, which was accompanied by a decreased number of macrophages in the BALF.

\section{Discussion}

An animal model of chronic respiratory infection was previously established by instilling agar beads containing $P$. aeruginosa into the rat trachea, where $P$. aeruginosa was slowly released out of the agar beads that remained in the trachea for a long period of time [14]. In the present study, rabbits were used as experimental animals. Unlike rats, rabbits have IL-8, which plays a key role in the evolution of many types of clinical diseases including lung [21]. The histological features of the rabbit model in the present study resembled clinical DPB; the recruitment of neutrophils was observed in the BALF. There were massive infiltrates of foamy alveolar macrophages and lymphocytes around the respiratory bronchioles, and these cells formed granulomatous lesions. It thus appears that the rabbit model can be used as a model of clinical DPB.

The therapeutic efficacy of EM for the treatment of clinical DPB has been ascribed to its anti-inflammatory properties, which include decreased production of inflammatory cytokines and the inhibition of neutrophil infiltration in BALF [7-11]. Correspondingly, the data showed that EM treatment inhibited the production of TNF- $\alpha$, IL- $1 \beta$ and IL- 8 , and the recruitment of neutrophils. More interestingly, it was found that administration of EM increased the recruitment of macrophages in BALF at an early phase (day 3), while decreasing it at a late phase (day 14). When the production of MCP-1 after $P$. aeruginosa inoculation was measured, MCP-1 levels in both BALF and lung were markedly increased after treatment with EM at the peak (day 1), at a time before the increased number of macrophages by EM (day 3). EM increased MCP1 mRNA expression in leukocytes recovered from BALF. These results suggest that increased production of MCP-1 on day 1 by EM is responsible for the increased number of macrophages in BALF on day 3 . As expected, MCP-1 blockade decreased the recruitment of macrophages in BALF.

Unlike the early phase, EM treatment decreased the number of macrophages in BALF in the late phase (day 14). This late phase of macrophage infiltration appeared to be independent of MCP-1, as anti-MCP1 antibodies did not affect the number of macrophages. The production of MCP-1 in both BALF and lung peaked on day 1 after $P$. aeruginosa inoculation, and decreased to the baseline level by day 7, while the influx of macrophages still increased after day 7 . This suggests that the late phase of macrophage infiltration is mediated by factor(s) other than MCP-1. TNF- $\alpha$ and IL-1 $\beta$, both of which were detected in this model, can induce many types of inflammatory mediators, including other $\mathrm{CC}$ chemokines [21 - 23]. It can be argued that such mediator(s) may be responsible for the later phase of macrophage influx and the production may be modulated by EM. It is further speculated that EM ameliorated the formation of granulomatous lesions on day 14 through the modulation of the mediator(s), not MCP-1. However, MCP-1 blockade abolished the protective effect of EM on formation of granulomatous lesions on day 14, without affecting the levels of TNF- $\alpha$ and IL-1 $\beta$. The data suggest that the increased production of MCP-1 by EM in the early phase 

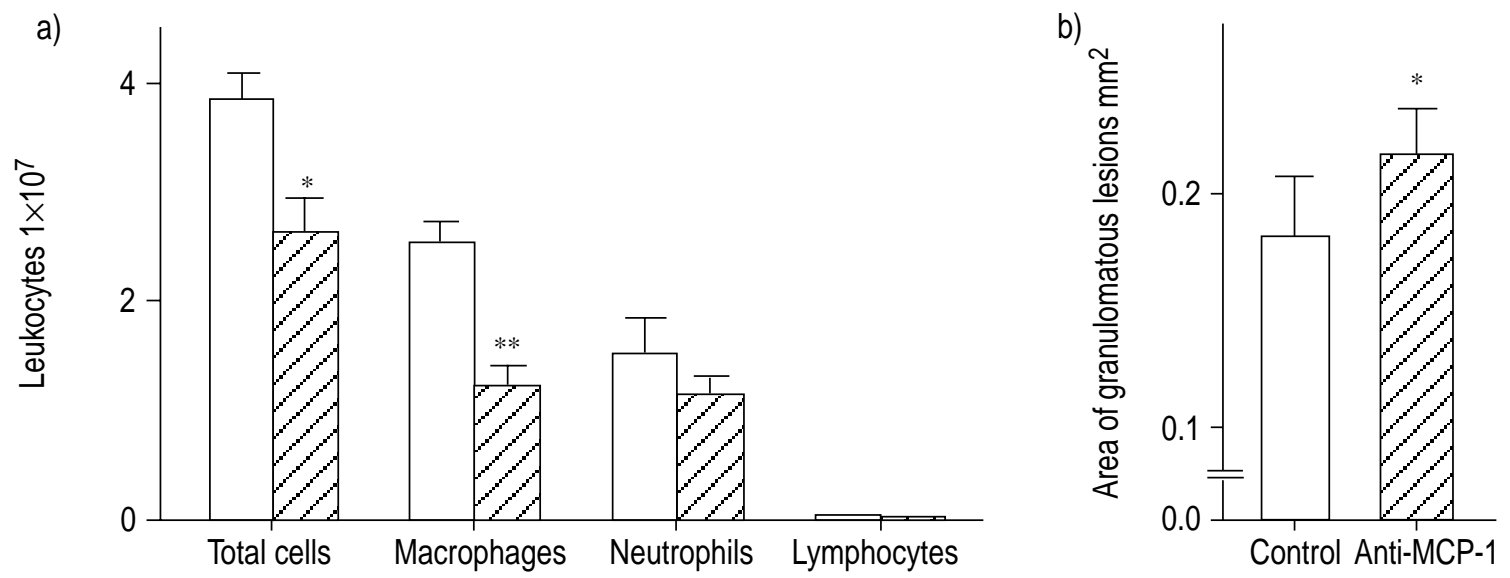

Fig. 6. - Effects of anti-monocyte chemoattractant protein (MCP)-1 antibodies on the numbers of leukocytes and the area of granulomatous lesion after treatment of rabbits with erythromycin. After $P$. aeruginosa inoculation, rabbits were treated with erythromycin plus either anti-MCP-1 antiserum (ש) or control serum ( $\square$ ). a) The number of leukocytes in bronchoalveolar lavage fluid were counted

paves the way towards interference in the formation of granulomatous lesions at the late phase.

The direct role of MCP-1 in regulating formation of granulomatous lesions in the present model is unclear. One possibility is that MCP-1 enhanced the phagocytic and killing activities of macrophages to $P$. aeruginosa MCP-1 can activate monocytes and cause lysozomal enzyme release [24] and $\mathrm{H}_{2} \mathrm{O}_{2}$ production [25], both of which are effector molecules for bacterial killing. Furthermore, earlier studies have demonstrated that an intraperitoneal administration of MCP-1 augmented the killing activity of peritoneal macrophage [26]. However, this is unlikely to be the mechanism at work in this model, because EM and an anti-MCP-1 antibody did not influence the number of viable bacteria recovered from the infected lung. Another possibility is that EM attenuates the granuloma formation via the modulation of TNF- $\alpha$, IL- $1 \beta$ and IL-8. These inflammatory cytokines may directly or indirectly affect granuloma formation. However, as discussed earlier, MCP-1 blockade abrogated the therapeutic effects of EM without altering the levels of these inflammatory cytokines. Although the function of MCP-1 has traditionally been viewed as a recruitment factor that regulate the accumulation of cells to the site of the response [21, 22], recent evidence has suggested that MCP-1 has broad activities in immune regulations. MCP-1 altered the cytokine balance, especially, by increasing the production of anti-inflammatory cytokines, as seen in an experimental endotoxaemia [27]. Other evidence suggested that MCP-1 could have a regulatory role on the immune system by altering cytokine production [28, 29]. Transforming growth factor (TGF)- $\beta$ can be induced by MCP-1 and may contribute to the modulation of granuloma formation in this study [30]. Since the present model features chronic pathological changes, it is possible that MCP-1 may influence immune responses in this model.

In conclusion, administration of erythromycin resulted in an increase in the number of macrophages in the bronchoalveolar lavage fluid, while decreasing the formation of pulmonary granulomatous lesions in the present model of chronic bronchiolitis. These events were accompanied by an increased production of monocyte chemoattractant protein-1. Neutralization studies showed that increased monocyte chemoattractant protein-1 production by erythromycin appeared to play a pivotal role in modulating the chronic phase of formation of granulomatous lesions, suggesting that erythromycin ameliorates pathological changes partly by up-regulating monocyte chemoattractant protein-1. Although the model employed in this study does not fully represent clinical diffuse panbronchiolitis, this novel erythromycin activity may be one of the mechanisms underlying its therapeutic efficacy in the treatment of clinical diffuse panbronchiolitis.

Acknowledgements. The authors would like to thank M. Kagayama and S. Kudoh for excellent technical assistance.

\section{References}

1. Myers JL, Colby TV. Pathologic manifestations of bronchiolitis, constrictive bronchiolitis, cryptogenic organizing pneumonia, and diffuse panbronchiolitis. Clinics Chest Med 1993; 14: 611-622.

2. Sugiyama Y. Difffise panbronchiolitis. Clin Chest Med 1993; 14: $765-772$

3. Kudoh S, Azuma A, Yamamoto M, Izumi T, Ando M. Improvement of survival in patients with diffuse panbronchiolitis treated with low-dose erythromycin. Am J Respir Crit Care Med 1998; 157: 1829-1832.

4. Kudoh S. Erythromycin treatment in diffuse panbronchiolitis. Curr Opin In Pulmon Med 1998; 4: $116-121$.

5. Nagai H, Shishido H, Yoneda R, Yamaguchi E, Tamura A, Kurashima A. Long-term low-dose administration of erythromycin to patients with diffuse panbronchiolitis. Respiration 1991; 58: 145-149. 
6. Koyama H, Geddes DM. Erythromycin and diffuse panbronchiolitis. Thorax 1997; 52: 915-918.

7. Iino $\mathrm{Y}$, Toriyama M, Kudo K, Natori Y, You A. Erythromycin inhibition of lipopolysaccharidestimulated tumor necrosis factor alpha production by human monocytes in vitro. Ann Otol Rhinol Laryngol 1992; S157: 16-20.

8. Akiyoshi H, Honda J, Nakahara S, et al. Mechanism of efficacy of erythromycin on diffuse panbronchiolitis-effect of erythromycin on cytokine mRNA expression in human whole blood model. Kansenshogaku Zasshi 1994; 68: 209-216.

9. Oishi K, Sonoda F, Kobayashi S, et al. Role of interleukin-8 (IL-8) and an inhibitory effect of erythromycin on IL-8 release in the airways of patients with chronic airway diseases. Infect Immun 1994; 62: $4145-4152$

10. Kadota J, Matsubara Y, Ishimatsu Y, et al. Significance of IL-1 beta and IL-1 receptor antagonist (IL-1Ra) in bronchoalveolar lavage fluid (BALF) in patients with diffuse panbronchiolitis (DPB). Clin Exp Immunol 1996; 103: $461-466$.

11. Sakito O, Kadota J, Kohno S, Abe K, Shirai R, Hara $\mathrm{K}$. Interleukin 1 beta, tumor necrosis factor alpha, and interleukin 8 in bronchoalveolar lavage fluid of patients with diffuse panbronchiolitis: a potential mechanism of macrolide therapy. Respiration 1996; 63: $42-48$.

12. Rollins BJ. Monocyte chemoattractant protein 1: a potential regulator of monocyte recruitment in inflammatory disease. Mol Med Today 1996; 2: 198-204.

13. Yoshimura T, Ueda A. Monocyte Chemoattractant Protein-1. In: Aggarwal BB, Gutterman JU, eds. Human Cytokines II: Handbook for Basic and Clinical Research. Oxford, Blackwell Scientific publications, 1996; $198-221$.

14. Cash HA, Woods DE, McCullough B, Johanson WG Jr, Bass JA. A rat model of chronic respiratory infection with Pseudomonas aeruginosa. Am Rev Respir Dis 1979; 119: 453-459.

15. Hirakata Y, Kaku M, Tomono K, et al. Efficacy of erythromycin lactobionate for treating Pseudomonas aeruginosa bacteremia in mice. Antimicrob Agents Chemother 1992; 36: $1198-1203$.

16. Matsukawa A, Miyazaki S, Maeda T, et al. Production and regulation of MCP-1 in lipopolysaccharideor monosodium urate crystal-induced arthritis in rabbits: Role of TNF $\alpha$, IL-1 and IL-8. Lab Invest 1998; 78: 973-985.

17. Fujiki M, Shinbori T, Suga M, Miyakawa H, Ando M. Role of $\mathrm{T}$ cells in bronchoalveolar space in the development of interstitial pneumonia induced by superantigen in autoimmune-prone mice. Am J Respir Cell Mol Biol 1999; 21: 675-683.
18. Matsukawa A, Ohkawara S, Maeda T, Takagi K, Yoshinaga M. Production of IL-1 and IL-1 receptor antagonist and the pathological significance in lipopolysaccharide-induced arthritis in rabbits. Clin Exp Immunol 1993; 93: 206-211.

19. Matsukawa A, Yoshimura T, Maeda T, Ohkawara S, Takagi K, Yoshinaga M. Neutrophil accumulation and activation by homologous IL-8 in rabbits. IL-8 induces destruction of cartilage and production of IL-1 and IL-1 receptor antagonist in vivo. J Immunol 1995; 154: $5418-5425$.

20. Flick DA, Gifford GE. Comparison of in vitro cell cytotoxic assays for tumor necrosis factor. J Immunol Methods 1984; 68: $167-175$.

21. Baggiolini M, Loetscher P, Moser B. Interleukin-8 and the chemokine family. Int J Immunopharmacol 1995; 17: $103-108$.

22. Graves DT, Jiang Y. Chemokines, a family of chemotactic cytokines. Crit Rev Oral Biol Med 1995; 6: $109-118$.

23. Dinarello CA. Role of pro- and anti-inflammatory cytokines during inflammation: experimental and clinical findings. J Biol Reg Homeos Agents 1997; 11: $91-103$.

24. Matsushima K, Larsen CG, DuBois GC, Oppenheim JJ. Purification and characterization of a novel monocyte chemotactic and activating factor produced by a human myelomonocytic cell line. J Exp Med 1989; 169: 1485-1490.

25. Rollins BJ, Walz A, Baggiolini M. Recombinant human MCP-1/JE induces chemotaxis, calcium flux, and the respiratory burst in human monocytes. Blood 1991; 78: $1112-1116$.

26. Nakano Y, Kasahara T, Mukaida N, Ko YC, Nakano M, Matsushima K. Protection against lethal bacterial infection in mice by monocyte-chemotactic and -activating factor. Infect Immun 1994; 62: 377-383.

27. Zisman DA, Kunkel SL, Strieter RM, et al. MCP-1 protects mice in lethal endotoxemia. J Clin Invest 1997; 99: $2832-2836$.

28. Karpus WJ, Lukacs NW, Kennedy KJ, Smith WS, Hurst SD, Barrett TA. Differential CC chemokineinduced enhancement of $\mathrm{T}$ helper cell cytokine production. J Immunol 1997; 158: 4129-4136.

29. Lukacs NW, Chensue SW, Karpus WJ, et al. C-C chemokines differentially alter interleukin-4 production from lymphocytes. Am J Pathol 1997; 150 $1861-1868$.

30. Gharaee-Kermani M, Denholm EM, Phan SH. Costimulation of fibroblast collagen and transforming growth factor beta 1 gene expression by monocyte chemoattractant protein-1 via specific receptors. J Biol Chem 1996; 271: 17779-17884. 